ARTICLE

DOI: 10.1057/s41599-018-0091-y

\title{
Moral narratives and mental health: rethinking understandings of distress and healthcare support in contexts of austerity and welfare reform
}

Felicity Thomas ${ }^{1}$, Lorraine Hansford ${ }^{1}$, Joseph Ford ${ }^{1}$, Katrina Wyatt ${ }^{1}$, Rosemarie McCabe ${ }^{1}$ \& Richard Byng ${ }^{2}$

\begin{abstract}
Associations between mental health and poverty are increasingly well established. Yet in neoliberally oriented contexts in which distress engendered through the everyday hardships of poverty is increasingly pathologised and medicalised, important questions are raised over the assumptions inherent within mental health policy and its implementation. Using the UK as a focus, this paper reviews and maps out key questions that require investigation in order to better understand the complex inter-relations between poverty and distress; explores how current paradigms might influence notions of individual responsibility and agency as well as health seeking behaviours; and examines the role of, and cultural and systemic expectations and constraints placed upon GPs as they respond to distress amongst patients from low-income communities. In so doing, we argue for recognition of the moral narratives that underpin both mental health care and processes of welfare reform, and call for an expansion of conventional notions of evidence-based healthcare to incorporate the understandings, experiences and priorities of people from low-income groups. We call for more detailed questioning and analysis of the interactions that lead to mental health diagnosis and treatment and better understanding of the relevance and effectiveness of current treatment options. As a central tenet of this, we argue for more flexible and nuanced healthcare responses that better reflect the dynamic and multi-faceted nature of poverty-related distress.
\end{abstract}

\footnotetext{
${ }^{1}$ University of Exeter, Exeter, UK. ${ }^{2}$ University of Plymouth, Plymouth, UK. Correspondence and requests for materials should be addressed to F.T. (email: f.thomas@exeter.ac.uk)
} 


\section{Introduction}

ince the publication of the 'Layard Report' (London School of Economics and Political Science Centre for Economic Performance's Mental Health Policy Group, 2006), the provision of effective mental health services has been firmly placed on the British Government agenda. Strategies such as No Health Without Mental Health' and more recent attempts to increase support services through the NHS 'Improving Access to Psychological Therapies (IAPT)' programme (NHS, 2015) demonstrate clearly how the UK government is seeking to move towards 'parity of esteem' between mental and physical health in terms of access to services, quality of care and allocation of resources (Parkin and Powell, 2017).

At the heart of these strategies is a commitment to make mental health provision available to all in society and to decrease the stigma and discrimination often associated with mental illness. In light of data suggesting that one in four people in the UK experience a mental health problem each year (Parkin and Powell, 2017), such intentions are clearly commendable. However, whilst these strategies set out reasonably clear plans for responding to mental ill health, there has been inadequate critique of the biomedical and diagnostic assumptions that underpin the evidence upon which such data and such strategies are based. This becomes deeply problematic when mental distress caused by challenging life circumstances is interpreted solely as clinical depression requiring medical intervention. At the same time, very little attention has been given to understanding how moralising narratives around personal responsibility intersect with broader social and structural circumstances to mediate people's understandings and experiences of mental (ill) health.

Given the known associations between poverty and mental health, it is pertinent to consider how, in the current context of economic austerity, strategies designed to reform the system of welfare support intersect with those aimed at supporting mental health and wellbeing. Certainly, it is increasingly recognised that the challenges of austerity, the hardships associated with recent welfare reforms and the associated introduction of Universal Credit can elicit various forms of mental distress amongst those affected (see, e.g., Cain, 2015; Citizen’s Advice Bureau, 2015). Yet, whilst it can be argued that distress is an unexceptional and expected reaction to difficult circumstances, the chances of this distress being diagnosed and treated as mental illness are now extremely high. If this means that people experiencing mental ill health are receiving appropriate support, then this may rightly be seen as an important step forward for healthcare parity. However, in situations where people's social standing and access to welfare support are increasingly and intrinsically connected to their health status and their ability to evidence sickness, it also raises a wide range of important ethical debates over the pathologisation and medicalisation of poverty and disadvantage.

Against this backdrop, this paper reviews and sets out key questions that need to be asked in order to deepen understanding of the ways that the dominant biomedical and moral narratives inherent within current mental health and welfare strategies may themselves be shaping experiences of, and responses to, mental health and wellbeing amongst low-income groups in the UK. The paper begins by examining how understandings and ways of responding to distress are socially determined within broader cultural and historical contexts, with particular focus on the role of biomedicine and psychiatry in shaping current ideas and languages around mental disorder, diagnosis and treatment. Attention is then given to the shifting inter-relationship between poverty and distress, and key questions raised around the ways that moralising narratives of responsibility intersect with pathologisation, medicalisation and notions of 'legitimate' welfare support. A more complete understanding here, we argue, requires much greater attention to be paid to the subjective perceptions and experiences of those within low-income communities whose voices are often marginalised within mental health research.

In reviewing dominant responses to mental distress, we emphasise the increasing pressure now placed upon GPs to provide for a range of medical and social support needs within consultations as other key services have been stripped back. Against this backdrop, we argue that it is important to understand how the systemic expectations and constraints placed upon GPs impact on their role as gatekeepers to diagnosis and treatment, and argue for much greater attention to be paid to both the wider structural context and the micro-scale interactions upon which consultation, diagnosis and treatment are based. Within this, we propose questions that need to be asked to better understand the relevance and effectiveness of existing systems of diagnosis and dominant treatment options, namely, antidepressant medications and talking therapies, for communities affected by poverty and deprivation.

\section{The pathologisation of distress}

At the heart of national and global mental health strategies lies an assumption that mental ill health is a pathological condition that can be understood and effectively dealt with through the use of demarcated diagnostic categories requiring distinct and specific forms of biomedical or therapeutic treatment. This is perhaps not surprising given the influence of biomedicine and the neurosciences in shaping understandings of mental health in recent decades, and recent calls by influential global institutions such as the World Health Organization to scale-up access to psychological and psychiatric treatments (WHO, 2013). Yet one only has to look to celebrated literary texts across time (think for example, Shakespeare's King Lear, de Cervantes' Don Quixote and Bronte's Mrs Rochester) to recognise that understandings of mental health and appropriate responses to the perceived afflictions of mental illness are socially determined within broader cultural and historical contexts. This is not to deny the reality of mental health issues within people's lives, but rather to acknowledge that if we are to comprehend the ubiquity and impact of mental distress within a contemporary UK context, then we need to recognise the ways that particular types of scientific knowledge and particular narratives of distress have been invested with meaning and authority, and as such, have the potential to become tools for use in the pursuit of broader political agendas.

A significant early influence on thinking around the pathologisation and medicalisation of mental distress can be found in Szasz's (1960) classic critique of psychiatry and his conceptualisation of mental illness as 'problems in living'. More recently, focus has been centred on the power and influence of diagnostic manuals, most notably the Diagnostic and Statistical Manual (DSM) and the International Classification of Diseases, with DSM-III, the 'bible of psychiatry' launched in 1980, attaining particular repute for setting in place crucial distinctions between what should be defined as 'normal' and what should not (Frances, 2013, p xii).

Rather than formulate a diagnosis around the background of the patient and their experiences and actions, these commonly used manuals prompt a diagnosis to be formulated if the patient has at least $\mathrm{x}$ number of symptoms from a given list within $\mathrm{y}$ weeks or months' (Brinkmann, 2014, p 635) depending on the specific diagnostic category at play. While the manuals themselves do not explicitly incorporate the 'biomedically diseased brain' concept, the surrounding discourse in psychiatric journals has, with some notable exceptions (see, e.g., Moncrieff et al., 2013; Tyrer, 2012), propagated the idea of an underlying pathology at 
the neuronal level. For some, the power and authority of this thinking is so great that it not only overlooks previously influential understandings of the human mind drawn from works of fiction, scientific investigation and philosophical and religious writings, but, through its innate and forceful implications around 'chemical imbalances' in the brain, has worked to reshape understandings of how the mind works and to challenge conceptions of agency, responsibility and free will (Whitaker, 2015).

Extending this work, others have analysed how psychiatry has transformed behaviours and emotions previously considered normal into mental 'disorder', in what Conrad (2007) has referred to as the 'pathologisation of everything'. Within this analysis, authors such as Conrad (2007 on ADHD) and Frances (2013 on PTSD) have demonstrated how behaviours that were once seen as part of normal social variation have become diagnosable as mental disorder. Others have examined how the authority of the DSM has expanded the boundaries of mental disorder through the reconfiguration of diagnostic categories so that, for example, emotions such as sadness have become recast as clinical depression (Horwitz and Wakefield, 2007); and how terms that have distinct meanings within psychiatry, for example, 'anxiety' and 'depression', have now become part of people's everyday vocabularies to explain behaviours, reactions and emotions that might once have been considered unremarkable (Brinkmann, 2014).

More recently, the lowering of thresholds for some diagnostic categorisations, and in particular, the removal of the bereavement exclusion clause from the criteria for major depressive disorder within DSM-5 have been a further cause for concern, leading Dowrick and Frances $(2013$, p 3) to lambast the recasting of grief as mental disorder as a 'medical intrusion into private emotions'. This, it is suggested, may in fact 'cultivate vulnerability' through encouraging people to feel depressed by experiences that were once regarded as routine (Furedi, 2004), and in turn, replace deeply embedded cultural rituals and emotive norms with a dubious and potentially stigmatising medical response.

Bringing an increasing array of 'conditions' and behaviours into the purview of psychiatry has invariably resulted in the massive increase in prescribing of antidepressant medications witnessed globally in recent years (OECD, 2015), accompanied by a parallel upturn, particularly in the global North, towards the use of psychological 'talking therapies'. These trends towards the pathologisation and medicalisation of what can be seen as understandable emotional norms raise important questions around the ways that we conceptualise various forms of mental distress. Given that poverty and deprivation are known to constitute key factors in the creation and exacerbation of mental distress, it is pertinent, particularly under existing conditions of austerity and welfare reform, to question current thinking around the intersections between poverty and mental ill-health and to examine the personal and societal implications of viewing poverty-induced distress as a pathological condition that necessarily requires medical intervention.

\section{Mental health, poverty and distress}

The association between poverty and mental ill health is now well established, with most explanations expounding a two-way process or a vicious cycle in which poverty may be seen to cause mental ill health, and mental ill health may be seen to lead to, or exacerbate poverty. There is consistent evidence for example, that people facing hunger and debt and living in poor or overcrowded housing, have high levels of mental health problems (Drentea and Reynolds, 2012); that possession of fewer material assets and poor employment is associated with depression (Rai et al., 2013); that people with mental illness are disproportionately represented amongst homeless populations (Fazel et al., 2014); and that children and adolescents with low socio-economic status are at higher risk of mental health problems than those in higher economic groups (Reiss, 2013).

A recent study found that the prevalence of mental health problems in England has increased markedly since the onset of economic recession since 2008, that increases were greatest in people with low levels of education and people out of work, and may be associated with welfare reforms and austerity measures (Barr et al., 2015). This is of particular concern when data indicate that overall poverty in the UK has risen in recent years and that this is set to rise further in the coming years without significant change to government policy (McGuinness, 2016).

Yet whilst research on deprivation and mental health can be useful in drawing attention to the potentially distressing effects of living in poverty, there is little conclusive evidence about the nature of the relationship between the factors at play (see Mills, 2015 for a detailed critique), nor, and as importantly, what it, and associated responses to this might mean in relation to people's lived experience and wellbeing. A key issue here relates to the ways in which mental health strategies, and much of the literature upon which they are based, are focused at the level of the individual psyche, framing mental health concerns as a pathological problem of the 'self (Busfield, 2011).

By considering mental health problems in a similar way to distinct bodily illnesses, there are two major implications for the ways that understandings of poverty and mental health are played out. First, a stance that is psychologically and behaviourally focused will inevitably reinforce a level of individualised blame and reiterate stereotypical assumptions about the behaviour of people living in deprived circumstances, leading to the reinscription of deficits-based thinking that sees distressed people who are living in poverty as somehow deficient and in need of 'correction' through medical or therapeutic intervention. Secondly, by pathologising individuals as having a distinct and categorisable 'defect' within their brain, mental health is very often viewed and treated within a disempowering apolitical vacuum, whilst the broader root causes of deprivation and social injustice that are known to sustain poverty and underpin the erosion of wellbeing become obscured (Shaw and Taplin, 2007; Friedli, 2013).

Moral narratives and systemic legitimacy. Critiquing this situation is not of course to deny the existence of intense distress and severe mental health issues within low-income communities. Rather, the central question examined here relates to what the pathologisation and medicalisation of poverty-related distress means for the health and wellbeing of people living in lowincome communities, and to recognise the ways that poverty may be exacerbating underlying vulnerabilities to stress. Understanding the implications of such circumstances for people's health and wellbeing is particularly apposite in the current economic climate, where notions of self and in particular, selfresponsibility, have been massively amplified through neoliberally oriented government policies to encourage the uptake of employment and to restrict welfare entitlements.

Inherent within such strategies, in popular contemporary media, and in encounters with key service providers such as job centres (see Friedli and Stearn, 2015), are moralising narratives that promote the idea that individuals and households facing challenging circumstances should have taken more responsibility for their health and wellbeing. Literature on the rise and consequences of libertarian paternalism has emphasised how the moral construction of 'good' and 'entrepreneurial' citizens (Crawford, 2006; Ayo, 2012) is evidenced amongst those who make choices informed by the guidance of expert authority to 
maximise personal and societal interests to improve and effectively 'manage' their health and wellbeing (Lupton, 1995; Leichter, 1997) whilst relieving the burden on the welfare system. This version of 'self-management' therefore encourages people to be active agents rather than passive recipients of 'care', be this in the form of health and/or welfare support (Ellis et al., 2017).

Whilst not contesting that this can be empowering, both in terms of self and political action, it is important to recognise the broader economic and narrative context in which such rhetoric is being pushed. Recent studies for example, suggest a hardening of attitudes amongst the British public against those in receipt of welfare support (NatCen, 2013), a factor that has increased feelings of shame, stigma and disassociation within low-income communities themselves (Shildrick and MacDonald, 2013). In a situation in which material deprivation and social disadvantage play a foundational role in emotional wellbeing, and when welfare entitlement is increasingly assessed via the possession of a legitimately certified 'disability', crucial questions arise concerning the ways that narratives around personal responsibility manifest, become embedded within everyday norms and expectations, and play out across diverse social groups, and in encounters with healthcare providers.

It is worth considering for example, how moralising narratives expounding the virtues of employment and the concurrent stigma associated with those seen as morally weak 'benefits scroungers' may be acting to push people towards seeking and taking up work; an issue that is especially pertinent when the government's 5 Year Forward View on Mental Health Taskforce (2016) very clearly states as one of its main goals, the need to support people to find or stay in work. Such circumstances may of course manifest in positive outcomes for those involved, both economically, and in terms of improving feelings of self-management, selfworth, agency and empowerment. Yet given that recent years have witnessed increasing levels of poverty within working households (JRF, 2016), and that work-related stress and poor mental wellbeing has been closely associated with the kinds of precarious and often low paid employment that is commonly available to those facing situations of hardship, it is also necessary to understand the role that such moralising narratives may be playing in pushing people towards situations which may ultimately reduce, rather than improve their quality of life.

At the same time, it is necessary to consider the extent to which the psychiatric reconfiguration of poverty that is inherent within the welfare legitimisation process encourages people to see and act upon themselves as if they were ill and in need of treatment (Furedi, 2004), and to consider how the medicalisation of poverty might encourage the incapacitation of individuals who may otherwise choose to work. To suggest that it does will undoubtedly have important implications for people's sense of responsibility, self-worth and resilience, as well as associated health and wellbeing, and can be seen as another form of moral narrative, in which people face a stark choice between 'taking accountability' and 'control' of their actions and emotions, or accepting a more passive, ill and 'defective' role in order to get support. As research undertaken in the United States has demonstrated, the latter option may also become an understandable survival strategy when being able to legitimately claim welfare support (through accepting a sick role and the medications and side effects that go with this) enables people to fulfil important social and familial roles (Hansen et al., 2014).

Pre-defining or circumscribing what counts as mental ill health amongst those living within circumstances of hardship, what matters to them, and how they (should) respond to this neglects consideration of people's agency, capabilities, and resourcefulness as well as the very real social and material constraints that affect their everyday lived experiences. Thus whilst health professionals are encouraged by clinical guidelines to emphasise diagnostic categories based upon pre-defined symptoms, patients are likely to understand their problems and seek to negotiate support for this within the unique biographical context that frames their lives. There is an urgent need therefore, to expand the evidence base away from the kind of research that reinforces a 'disembodied psychology' (Freidli and Stearn, 2015), and towards an examination of how people in low-income communities themselves conceptualise, experience and explain their distress within the broader biographical and situated context of their lives-both to those within their immediate social networks, and to those, such as GPs, whose assistance they seek. These kinds of subjectively informed insights would then enable deeper exploration around the ways that experiences of distress may be shaped or amplified through the moral narratives that underpin notions of deservingness within the contemporary welfare state and would help raise much needed debate around the integrity and ethics of sickness-based welfare support.

\section{Responding to distress}

Understanding how people in low-income communities conceptualise, talk about and embody mental distress quite clearly has significant implications for the ways that they seek (or avoid) support in response to it. Research has shown for example, that many people in low-income communities face a range of culturally determined issues that deter them from seeking help, and that this is particularly evident amongst men (Clement et al., 2015). Other studies have stressed the value of informal social networks that enable people to share their experiences amongst trusted networks (Brown et al., 2014), and more work is needed to better understand how such mechanisms function and how they can be effectively fostered and supported.

Despite the challenges people face in seeking support, it is clear from national consultation data that many people from lowincome communities do at some point in time turn to health professionals to seek support for mental distress. This may indicate that the patient understands their distress through a medical framework, and accepts that they require some form of medically-oriented treatment. However, in a situation in which large-scale resource cuts in the UK voluntary sector mean that GPs are often the only place left for people to go to for help, and where GPs act as the main conduit for the administration of sick notes (now tellingly named 'fitness for work' notes) needed by people to evidence their ill health (Wheat et al., 2015), the picture is often far from clear-cut.

A better understanding is needed therefore of both the expectations that patients from low-income backgrounds place on their encounters with health providers for mental distress, and the ways that health providers seek to respond to this within the broader systemic, logistical and cultural boundaries in which they work. Addressing these knowledge gaps calls for mental health research to be broadened away from an objective clinical gaze to encompass the interpretive forms of understanding that can be gained through qualitative methods which foreground the perspectives of, and exchanges between, patients and health care providers.

GP-patient interactions. Existing information on the experiences of general population groups (see, e.g., Karasz et al., 2012) suggest that physician decision-making is associated with the type of narrative the patient presents with. Yet conversations between doctors and patients as they relate to mental distress have been little investigated, and there is a scarcity of knowledge around the kinds of verbal and gestured interactions and informational exchange that takes place during a consultation that may 
influence both physician and patient decision making around presentation, diagnosis and treatment.

The dearth of in-depth research within this area is an important lacuna since health care consultations are infused with 'moral work' as patients seek to justify their attendance in order to gain a legitimised sick role (Seale et al., 2013; Wheat et al., 2015) and because GPs are now required to act as gatekeepers to treatments aimed at alleviating mental distress. Key lines of enquiry therefore need to consider how moral narratives around personal responsibility are played out within primary care consultations, and the role played by the patient and the GP in the negotiation and production of diagnosis and treatment. Within this, it will be important to consider how the need to evidence sickness to redeem work or welfare entitlements or social status might intersect both with widely accepted notions of distress and responsibility and with the kinds of decision algorithms and tools provided within clinical guidelines for diagnosing depression and anxiety (e.g. PHQ-9, GAD-7), and subsequent treatment and onward patient 'disposal' (Byng, 2012).

At the heart of this debate is the necessity to consider how particular forms of biomedical and narrative evidence are drawn upon to shape the direction of the consultation, and to assess the status of a patient's own understandings and subjectively defined experiences and needs against the broader clinical frameworks within which GPs in the UK are currently obliged to work.

Recognising how different kinds of treatment are sought, negotiated and administered and how such interventions are then experienced by those concerned would also provide much needed information on the perceived relevance and effectiveness of available support, and the ways that these play out across diverse and temporal contexts of distress. This is important when the main types of mental health support currently available to respond to what are often extremely complex, multifaceted situations, are limited to two fairly stark and contentious treatment options, namely, antidepressant medications and/or talking therapies.

Antidepressant medications. The number of antidepressant items prescribed in the UK has more than doubled in the last decade. In 2016, there were 64.7 million antidepressant items dispensed, 33.7 million (108.5\%) more than in 2006, when there were 31.0 million (NHS Digital, 2017). Recent analysis demonstrates disproportionately high levels of prescribing and use of psychiatric drugs within low-income communities (EXASOL, 2017; Anderson et al., 2009). One reading of this may of course be that mental health services have successfully challenged the inverse care law, by ensuring that treatments are available to all. Yet whilst not disputing that the use of such medications may be beneficial to some patients, mounting evidence now demonstrates that such drugs have little or no effect in cases of mild depression, and indeed, carry risks associated with harmful side effects, including increased suicidal thinking, as well as the potential for adverse interactions with drugs prescribed for other health issues (Gøtzsche, 2015).

In any analysis of medication prescribing practice, it is of course necessary to recognise the very limited options available to GPs who are increasingly dealing with complex cases within the bounds of restricted time and financial resourcing. However, it is also worth considering how the cultures of prescribing embedded within primary health care practice may be influenced not only by structural and resourcing constraints, but by preconceptions and biases around the perceived behaviour and needs of patients from low-income communities. Research in other areas of medicine has demonstrated how embedded cultures of working practice can influence not only levels of prescribing, but also the types of medications administered to particular social groups. Zaharan et al. (2014) for example, found that newer, more costly treatments for diabetes were being prescribed to patients from higher socio-economic groups in Ireland, despite all patients in the study receiving free prescriptions. Existing data from the UK already suggest that GP characteristics such as age and place of training can influence the likelihood of antidepressant prescribing practice (Spence et al., 2013), and it is important that research in this area is prioritised so that elements of effective as well as potentially detrimental practice can be better understood. At the same time, any attempt to gain insight into prescribing practice requires greatly improved understanding of what patients from diverse social groups expect when they seek support from a GP, and how these expectations are conveyed and in turn, interpreted by health professionals.

Questions around the intersections between clinical prescribing, resourcing, and patient expectation and agency are also relevant to discussions on the problematic nature of both adherence and long-term antidepressant use. Research has shown that a high number of patients discontinue treatment early (Sansone and Sansone, 2012), yet little is known about why, how this impacts on wellbeing, or whether these people return to their GPs for further support. On the other hand, an increasing body of evidence around iatrogenesis suggests that in many cases, longterm use of psychiatric medicines not only exacerbates existing mental health conditions, but may also trigger new complaints. Data from a recently published 20 year study for example, show that at each follow-up assessment, people who had taken antipsychotic drugs were significantly more likely to display psychotic symptoms than those who had never taken medication (Harrow et al., 2014), whilst Kirsch et al. (2008) has argued that antidepressants not only have limited effectiveness over placebos but may also induce 'biological vulnerability' that makes people more prone to depression in their future lives. The diverse and insightful case stories followed by Whitaker's (2015) study in the United States similarly describe the disturbing possibility that long term use of psychiatric drugs can significantly aggravate what started off as relatively minor episodes of mental ill health.

This is important when estimates suggest that half of all people on antidepressants in the UK have been taking them for 2 years or more (Kendrick, 2015), and that nationwide, a third of all people taking them long-term have no clinical reason to continue, and could try stopping treatment (Cruickshank et al., 2008). In a situation in which GPs are discouraged by clinical guidelines to reassess treatment decisions except in cases when side effects are palpably detrimental to patient health, when health providers are massively time-constrained by cuts to resourcing, and when people may assume that they are expected to continue treatment unless otherwise advised, the likelihood of people remaining on antidepressants in the long term becomes increasingly apparent.

Research that examines the inclination and willingness of patients from diverse social groups to contest the status quo, as well as the experiences and motivations of GPs who seek to provide alternative ways of working within consultations could therefore illuminate important insights around factors influencing patient expectation, experience and agency and around the impact of alternative health care and support for patient health and wellbeing outcomes. Given the known issues associated with withdrawal from antidepressants (see Cartwright et al., 2016), there is also a need to address the startling lack of information on the kinds of support available to people from diverse social groups who wish to discontinue treatment.

Talking therapies. Alongside the accelerated use of antidepressant medications, recent years have seen the UK 
Government commit strongly to non-medical interventions through the Improving Access to Psychological Therapies (IAPT) programme. Launched in 2008, this programme claims to have supported over 900,000 patients a year with plans to expand this to upward of 1.5 million a year by 2020 . Whilst existing evidence suggests that IAPT has increased provision of treatments that may be effective for some people who have low mood or anxiety (see Clark, 2011), there has been relatively little analysis of how it is sought, accessed and experienced across diverse social groups, or how it impacts on people who are seeking help for distress which may be caused primarily by their broader life situation and past traumas.

Understanding here is especially important when such services are currently delivered and evaluated through a 'one-size fits all' approach that disregards social and economic variation in need and provision in order to meet standardised 'targets around 'recovery'. Better understanding of the effectiveness of psychological therapies is also critical given that they are explicitly identified within the Government's 5 Year Forward View on Mental Health Taskforce (2016) as a key tool in its broader efforts to help people to find or stay in employment, with little obvious regard for the precarious, irregular and potentially harmful nature of most work available to those in low-income communities in economically depressed areas of the country.

Research demonstrates that rates of non-attendance within IAPT are high and have complex causes (Marshall et al., 2016). What little evidence exists around experiences of IAPT amongst people from low-income groups suggests that embedded cultural dispositions often play a key role in deterring them from attending and benefiting from these kinds of therapeutic support service (Holman, 2014). If this is the case, such findings lie at odds with the commonly accepted requirement for people to selfrefer to IAPT as a 'first step to recovery', and may also throw question on the much heralded potential of recent alternative (not yet mainstream) approaches e.g. the Power Threat Meaning Framework, that aim to take a more holistic approach to addressing distress (Johnstone and Boyle, 2018). It is also worth considering that those acting as their gatekeepers may bestow different treatments with differing expectations relating to patient agency and responsibility. Research within the field of HIV for example, has demonstrated how access to anti-retroviral therapy has been mediated by both confessional practices and particular notions of therapeutic citizenship that carry moralising messages around deservingness and adherence (Fung, 2014; Mattes, 2012). Whether GPs draw distinctions between the prescribing of what may be seen by some as 'quick fix' antidepressant medications that can be administered to citizens deemed as 'passive' and 'deficit', and the prescribing of talking therapies to those considered capable of exerting the labour and commitment deemed necessary in order to 'help themselves', are important areas for exploration that would help provide much needed insight into the availability and take up of IAPT.

Understanding co-morbidities. People in low-income communities commonly live with a range of coexisting health and wellbeing issues that cross-cut mental, physical and social spheres. Yet the dominance of antidepressant prescribing and talking therapies as a response to distress, demonstrates clearly how current diagnostic and treatment frameworks assume a separation of problems that are seen to require quite distinct treatment pathways. What this means in practice in terms of patient and health provider experience has been little investigated, particularly in the field of mental health, yet has direct implications not only for individual health and wellbeing outcomes, but for broader debates around the structure and economics of healthcare provision. Seeking greater understanding of the possibilities of establishing practice that takes a more holistic approach to understanding the complex interplay between coexisting physical, mental and social problems (as is set out as a key aim by the NHS's Implementing the 5 Year Forward View for Mental Health, 2017) is therefore vital if the realities of people's lives are to be more effectively supported in the coming years.

\section{Conclusions}

The provision of effective mental health support for all in society is now a firmly stated aim of the British Government. At the heart of the mental health strategies being rolled out is a desire to decrease stigma and discrimination and to move towards parity of esteem between mental and physical health. Yet, as this review has argued, current programmes of mental health support are intrinsically bound up with wider political agendas and with moralising narratives relating to welfare, employment, responsibility and deservingness, thus posing a clear paradox for the realisation of meaningful and effective care and support for some of the UK's most vulnerable populations.

Indeed, we have argued that the dominant psychiatric and moral narratives inherent within current mental health and welfare strategies may themselves be shaping experiences of, and responses to mental health and wellbeing in often negative and potentially harmful ways. This includes the potential for the psychiatric reconfiguration of poverty inherent within the welfare legitimisation process to encourage people experiencing distress to see and act upon themselves as if they were ill and in need of treatment, as well as the uptake of potentially harmful medications that may result from this. And whilst some people may well ultimately benefit from the current push towards employment and away from welfare, it is likely that many others feel forced to take up employment that may ultimately leave them in a situation that reduces rather than improves their mental, and often physical, wellbeing and quality of life.

Understanding how these issues play out, we have argued, requires research that moves away from what may be an overly 'clinical', protocol-focused gaze to consider the perceptions and lived experiences of those within low-income communities whose voices are too often marginalised within mental health research. Achieving this requires that attention be paid to understanding how people in low-income communities themselves conceptualise, experience and explain their distress within the broader biographical and situated context of their lives.

It will also be important to understand how some individuals, often without support from health services, can overcome adversity through personal action, sometimes of a political nature. Equally, we must understand how traumas inflicted from within communities, often by men on women and children, interact with economic causes of mental distress. Such research is likely to be best achieved through participatory research approaches that ensure that the aims, objectives, delivery and interpretation of data are negotiated with participants. Opening up space for marginalised voices, and helping to identify whether experiences of, as well as responses to, distress can be framed and articulated in a manner that may be more in line both with subjectively defined needs and the broader social and structural contexts in which they are encountered, also means acknowledging that languages of mental distress extend beyond the dominant narratives of psychiatry and take on board the complex interplay between mental, physical and social circumstances. Such a project could incorporate an understanding of how the brain's function is affected by distress stemming from structural adversity, trauma and loss, as well as seek to identify a broad set of interventions that those affected would deem supportive. 
At the same time, we have argued that it is vital that research on mental health seeks better understanding of the ways that cultures of diagnosis and prescription become embedded within clinical practice, and we have emphasised the need for better understanding of the systemic expectations and constraints that currently frame primary health care. This, we have argued, is particularly vital when GPs are increasingly located at the junctures of mental health care delivery and (albeit often unwillingly) the gatekeeping of sickness-based welfare support.

Received: 26 January 2018 Accepted: 13 March 2018

Published online: 10 April 2018

\section{References}

Ayo N (2012) Understanding health promotion in a neoliberal climate and the making of health conscious citizens. Crit Public Health 22(1):99-105

Anderson S, Brownlie J, Given L (2009) Therapy culture? Attitudes towards emotional support in Britain. In: Park A, Curtice J, Thomson K, Phillips M, Clery E (eds) British social attitudes: the 25th report. Sage, London, pp $155-172$

Barr B, Kinderman P, Whitehead M (2015) Trends in mental health inequalities in England during a period of recession, austerity and welfare reform 2004 to 2013. Social Sci Med 147:324-331

Brinkmann S (2014) Languages of suffering. Theory Psychol 24(5):630-648

Brown JSL, Evans-Lacko S, Aschan L, Henderson MJ, Jatch SL, Hotopf M (2014) Seeking informal and formal help for mental health problems in the community: a secondary analysis from a psychiatric morbidity survey in South London. BMC Psychiatry 14:275

Busfield J (2011) Mental Illness. Polity, London

Byng R (2012) Recognition of distress and depression in primary care: how far should we go? Fam Pract 29(1):1-2

Cain R (2015) Work at all costs? The gendered impact of universal credit on loneparent and low-paid families. Engenderings. Blog Entry, London School of Economics. http://eprints.lse.ac.uk/78596/. accessed 15 Dec 2017

Cartwright C, Gibson K, Read J, Cowan O, Dehar T (2016) Long-term antidepressant use: patient perspectives on benefits and adverse effects. Patienet Prefer Adherence 10:1401-1407

Citizen's Advice Bureau (2015) Waiting for credit: the delivery of universal credit as experienced by citizen's advice clients in England and Wales. Citizen's Advice Bureau. http://www.boltoncab.co.uk/Global/Waiting\%20for\%20Cre dit\%20PDF\%20final\%20(003)\%20Nov\%2020.15.pdf. accessed 12 Dec 2017

Clark DM (2011) Implementing NICE guidelines for the psychological treatment of depression and anxiety disorders: the IAPT experience. Int Rev Psychiatry 23(4):318-327

Clement S, Schauman O, Graham T, Maggioni F (2015) What is the impact of mental health-related stigma on help-seeking? A systematic review of quantitative and qualitative studies. Psychol Med 45(1):11-27

Conrad P (2007) The medicalization of society: on the transformation of human conditions into medical disorders. John Hopkins University Press, Baltimore

Crawford R (2006) Health as a meaningful social practice. Health 10(4):401-420

Cruickshank G, MacGillivray S, Bruce D, Mather A, Matthews K, Williams B (2008) Cross-sectional survey of patients in receipt of long-term repeat prescriptions for antidepressant drugs in primary care. Ment Health Fam Med 5(2):105-109

Dowrick C, Frances A (2013) Medicalising unhappiness: new classification of depression risks more patients being put on drug treatment from which they will not benefit. Br Med J 347:f7140

Drentea P, Reynolds JR (2012) Neither a borrower nor a lender be: the relative importance of debt and SES for mental health among older adults. J Aging Health 24(4):673-695

Ellis J, Boger E, Latter S, Kennedy A, Jones F, Foster C, Demain S (2017) Conceptualisation of the 'good' self-manager: a qualitative investigation of stakeholder views on the self-management of long-term health conditions. Social Sci Med 176:25-33

EXASOL (2017) EXASOL analyzes: research shows that over 64, prescriptions of antidepressants are dispensed per year in England. Visualised for the first time. EXASOL. https://www.exasol.com/en/company/newsroom/news-and-press/ 2017-04-13-over-64-million-prescriptions-of-antidepressants-dispensed-peryear-in-england/. accessed 6 Dec 2017

Fazel S, Geddes JR, Kushel M (2014) The health of homeless people in high-income countries: descriptive epidemiology, health consequences, and clinical and policy recommendations. Lancet 384(9953):1529-1540
Frances A (2013) Saving normal: an insider's revolt against out-of-control psychiatric diagnosis, DSM-5, big pharma, and the medicalization of ordinary Life. HarperCollins, New York

Friedli L (2013) What we've tried, hasn't worked: the politics of assets based public health. Crit Public Health 23(2):131-145

Friedli L, Stearn R (2015) Positive affect as coercive strategy: Conditionality, activation and the role of psychology in UK government workfare programmes. Med Humanit 41:40-47

Fung D (2014) Migrant narratives, chronicity and HIV in London. PhD thesis, Edinburgh Research Archive

Furedi F (2004) Therapy culture: cultivating vulnerability in an uncertain age. Routledge, London

Gøtzsche PC (2015) Does long term use of psychiatric drugs cause more harm than good? Br Med J 350:h2435

Hansen H, Bourgois P, Drucker E (2014) Pathologizing poverty: New forms of diagnosis, disability, and structural stigma under welfare reform. Social Sci Med 103:76-83

Harrow M, Jobe TH, Faull RN (2014) Does treatment of schizophrenia with antipsychotic medications eliminate or reduce psychosis? A 20-year multifollow-up study. Psychol Med 44(14):3007-3016

Holman D (2014) What help can you get talking to somebody? Explaining class differences in the use of talking treatments. Sociol Health Illn 36(4):531-548

Horwitz AV, Wakefield JC (2007) The Loss of Sadness: How psychiatry transformed normal sorrow into depressive disorder. New York: Oxford University Press

Johnstone L, Boyle M, Cromby J, Dillon J, Harper D, Kinderman P, Longden E, Pilgrim D, Read J (2018) The power threat meaning framework: towards the identification of patterns in emotional distress, unusual experiences and troubled or troubling behavior, as an alternative to functional psychiatric diagnosis. British Psychological Society, Leicester

JRF (2016) Monitoring poverty and social exclusion 2016. https://www.jrf.org.uk/ report/monitoring-poverty-andsocial-exclusion-2016. accessed 3 Jan 2018

Karasz A et al. (2012) What we talk about when we talk about depression: doctorpatient conversations and treatment decision outcomes. Br J Gen Pract 62 (594):55-63

Kendrick T (2015) Long-term antidepressant treatment: time for a review. Prescriber 26(19):7-10

Kirsch I, Deacon BJ, Huedo-Medina TB, Scoboria A, Moore TJ, Johnson BT (2008) Initial severity an antidepressant benefits: a meta-analysis of data submitted to the food and drug administration. PLoS Med 5(2):e45. https://doi.org/ 10.1371/journal.pmed.0050045

Leichter HM (1997) Lifestyle correctness and the new secular morality. In: Brandt AM, Rozin P (eds) Morality and Health. Routledge, New York, pp 359-378

London School of Economics and Political Science. Centre for Economic Performance's Mental Health Policy Group (2006) The depression report: a new deal for depression and anxiety disorders. LSE, London

Lupton D (1995) The imperative of health: public health and the regulated body. Sage, London

Marshall D, Quinn C, Child S, Shenton D, Pooler J, Forber S, Byng R (2016) What IAPT services can learn from those who do not attend. J Ment Health 25 (5):410-415

Mattes D (2012) I am also a human being!: antiretroviral treatment in local moral worlds. Anthropol Med 19(1):75-84

McGuinness F (2016) Poverty in the UK: statistics. Briefing Paper No. 7096. House of Commons Library, London, UK

Mental Health Taskforce (2016) The five year forward view for mental health Mental Health Taskforce. https://www.england.nhs.uk/wp-content/uploads/ 2016/02/Mental-Health-Taskforce-FYFV-final.pdf. accessed 5 Nov 2017

Mills C (2015) The psychiatrization of poverty: rethinking the mental healthpoverty nexus. Social Personal Psychol Compass 9(5):213-222

Moncrieff J, Cohen D, Porter S (2013) The psychoactive effects of psychiatric medication: the elephant in the room. J Psychoact Drugs 45(5):409-415

NatCen (2013) Public attitudes to poverty and welfare 1983-2011. NatCen. http:// www.natcen.ac.uk/our-research/research/public-attitudes-to-poverty-andwelfare-1983-2011/. accessed 11 Nov 2017

NHS Digital (2017) Prescriptions dispensed in the community, statistics for England-2006-2016[PAS]. NHS. http://digital.nhs.uk/catalogue/PUB30014. accessed 2 Jan 2018

NHS (2015) Improving access to psychological therapies. NHS. http://www.iapt. nhs.uk. accessed online 3 Oct 2017

OECD (2015) Health at a glance 2015: OECD indicators. OECD Publishing, Paris

Parkin E, Powell T (2017) Mental health policy in England. Briefing Paper No. CBP 07547. House of Commons Library, London, UK

Rai D, Zitko P, Jones K, Lynch J, Araya R (2013) Country- and individual-level socioeconomic determinants of depression: multilevel cross-national comparison. Br J Psychiatry 202(3):195-203

Reiss F (2013) Socioeconomic inequalities and mental health problems in children and adolescents: a systematic review. Social Sci Med 90:24-31 
Sansone RA, Sansone LA (2012) Antidepressant adherence: are patients taking their medications? Innov Clin Neurosci 9(5-6):41-46

Seale C, Rivas C, Al-Sarraj H, Webb S, Kelly M (2013) Moral mediation in interpreted health care consultations. Soc Sci Med 98:141-148

Shaw I, Taplin S (2007) Happiness and mental health policy: a sociological critique. J Ment Health 16(3):359-373

Shildrick T, MacDonald R (2013) Poverty talk: how people experiencing poverty deny their poverty and why they blame 'the poor'. Sociol Rev 61 (2):285-303

Spence R, Roberts A, Ariti C and Bardsley M (2013) Focus on antidepressant prescribing: trends in the prescribing of antidepressants in primary care. QualityWatch. http://www.qualitywatch.org.uk/sites/files/qualitywatch/field/ field_document/140528_QualityWatch_Focus\%20on_antidepressant $\%$ 20prescribing_0.pdf. accessed online 5 Sept 2017

Szasz MD (1960) The myth of mental illness. Am Psychol 15:113-118

Tyrer P (2012) The end of the psychopharmacological revolution. Br J Psychiatry 201(2):1868

Wheat HC, Barnes RK, Byng R (2015) Practices used for recommending sickness certification by general practitioners: a conversation analytic study of UK primary care consultations. Social Sci Med 126:48-58

Whitaker R (2015) Anatomy of an epidemic: magic bullets, psychiatric drugs, and the astonishing rise of mental illness in America. Broadway Books, New York

WHO (2013) Mental Health Action Plan. WHO, Geneva, pp 2013-2020

Zaharan NL, Williams D, Bennett DWK (2014) Prescribing of antidiabetic therapies in Ireland: 10 year trends 2s003-2012. Ir J Med Sci 183(2):311-318

\section{Data availability}

Data sharing is not applicable to this paper as no datasets were analysed orgenerated.

\section{Acknowledgements}

This paper is based on research funded by the UK's Economic and Social Research Council, grant ref. ES/N018281/1.

\section{Additional information}

Competing interests: The authors declare no competing financial interests.

Reprints and permission information is available online at http://www.nature.com/ reprints

Publisher's note: Springer Nature remains neutral with regard to jurisdictional claims in published maps and institutional affiliations.

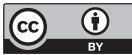

Open Access This article is licensed under a Creative Commons Attribution 4.0 International License, which permits use, sharing, adaptation, distribution and reproduction in any medium or format, as long as you give appropriate credit to the original author(s) and the source, provide a link to the Creative Commons license, and indicate if changes were made. The images or other third party material in this article are included in the article's Creative Commons license, unless indicated otherwise in a credit line to the material. If material is not included in the article's Creative Commons license and your intended use is not permitted by statutory regulation or exceeds the permitted use, you will need to obtain permission directly from the copyright holder. To view a copy of this license, visit http://creativecommons.org/ licenses/by/4.0/.

(C) The Author(s) 2018 